\title{
Myofibril Degeneration Caused by Tropomodulin Overexpression Leads to Dilated Cardiomyopathy in Juvenile Mice
}

\author{
Mark A. Sussman, ${ }^{\star}$ Sara Welch, ${ }^{\star}$ Natalie Cambon, ${ }^{\star}$ Raisa Klevitsky, ${ }^{,}$Timothy E. Hewett, ${ }^{\star}$ Robert Price, ${ }^{\S}$ Sandra A. Witt, ${ }^{\ddagger}$ \\ and Thomas R. Kimball \\ The Children’s Hospital and Research Foundation, *Division of Molecular Cardiovascular Biology and ${ }^{\ddagger}$ Division of Cardiology, \\ Cincinnati, Ohio 45229; and ${ }^{\S}$ Department of Developmental Biology and Anatomy, University of South Carolina, School of Medicine, \\ Columbia, South Carolina 29208
}

\begin{abstract}
Loss of myofibril organization is a common feature of chronic dilated and progressive cardiomyopathy. To study how the heart compensates for myofibril degeneration, transgenic mice were created that undergo progressive loss of myofibrils after birth. Myofibril degeneration was induced by overexpression of tropomodulin, a component of the thin filament complex which determines and maintains sarcomeric actin filament length. The tropomodulin cDNA was placed under control of the $\alpha$-myosin heavy chain gene promoter to overexpress tropomodulin specifically in the myocardium. Offspring with the most severe phenotype showed cardiomyopathic changes between 2 and 4 wk after birth. Hearts from these mice present characteristics consistent with dilated cardiomyopathy and a failed hypertrophic response. Histological analysis showed widespread loss of myofibril organization. Confocal microscopy of isolated cardiomyocytes revealed intense tropomodulin immunoreactivity in transgenic mice together with abnormal coincidence of tropomodulin and $\alpha$-actinin reactivity at $Z$ discs. Contractile function was compromised severely as determined by echocardiographic analyses and isolated Langendorff heart preparations. This novel experimentally induced cardiomyopathy will be useful for understanding dilated cardiomyopathy and the effect of thin filament-based myofibril degeneration upon cardiac structure and function. (J. Clin. Invest. 1998. 101:51-61.) Key words: myocardial diseases • cardiomyopathy • ventricular dysfunction • myofibrils • myocardium
\end{abstract}

\section{Introduction}

Congestive heart failure is among the most important public health problems in cardiovascular medicine and the most rapidly growing cardiovascular disorder in the United States (1). Heart failure is a major cause of disability and morbidity with

Address correspondence to Mark A. Sussman, The Children's Hospital and Research Foundation, Division of Molecular Cardiovascular Biology, Room 3033, 3333 Burnet Avenue, Cincinnati, OH 45229. Phone: 513-636-7145; FAX: 513-636-8966; E-mail: sussman@heart. chmcc.org

Received for publication 10 July 1997 and accepted in revised form 29 October 1997.

J. Clin. Invest.

(C) The American Society for Clinical Investigation, Inc. 0021-9738/98/01/0051/11 \$2.00

Volume 101, Number 1, January 1998, 51-61

http://www.jci.org over $\$ 8$ billion spent annually for patient care (1). The most common form of heart failure associated with loss of systolic function is dilated cardiomyopathy. A substantial amount of clinical data has been gathered regarding the epidemiology of heart failure and dilated cardiomyopathy (2). These studies show that new treatment modalities have only reduced heart failure by an average of $14 \%$ over the last $40 \mathrm{yr}$. Heart failure remains highly lethal, particularly for patients with severe symptoms and left ventricular $(\mathrm{LV})^{1}$ dysfunction. Despite the prevalence and severity of dilated cardiomyopathy, relatively little is known regarding etiology. The "three Ds" of cardiac failure pharmacotherapy (digitalis, diuretics, and vasodilators) have prolonged survival significantly (3). Such treatment primarily relieves symptoms associated with heart failure, but not the underlying cause. In part due to drug therapy, the course of heart failure no longer progresses predictably over years from mild to severe disease as was true $30-50 \mathrm{yr}$ ago (4). Today, advanced symptoms can develop in weeks to months with sudden death possible at any time. Most importantly, the prevalence of dilated cardiomyopathy has steadily increased over the last $20 \mathrm{yr}$.

Loss of myofibrils is the most obvious structural change in dilated cardiomyopathy (5) and sarcomeric disarray is characteristic of failing hearts (6). Features of myocardium from these patients include increased myocardial mass and ventricular chamber size, myocyte and myofibril disarray (7), and fatty infiltration or mononuclear cell invasion (8). Changes seen in endomyocardial biopsies from patients of dilated cardiomyopathy (9) include: loss of contractile material, enlargement of nuclei, enlargement of extracellular space, increased glycogen deposition and thinning of myocardial walls, focal hypertrophy, myocyte atrophy, and accumulation of collagen in perivascular and interstitial areas. The number and severity of pathological changes in human samples shows marked individual variation. Understanding the effect of myofibril degeneration upon cardiac function is a prerequisite for improving our ability to assess and potentially mitigate a wide variety of cardiomyopathies.

Accurate control of thin filament length in sarcomeres is critical for proper function of the contractile apparatus, which in turn depends upon consistent length specification and effec-

1. Abbreviations used in this paper: Ao, aortic root diameter; $+d P / d t$, the first derivative of the increase in left ventricular systolic pressure with respect to time; $-d P / d t$, the first derivative of the decrease in left ventricular systolic pressure with respect to time, a measure of relaxation; GAPDH, glyceraldehyde 3-phosphate dehydrogenase; LV, left ventricular; LVED, LV end-diastolic dimension; MHC, myosin heavy chain; MLC-2v, myosin light chain (ventricular isoform); PTU, 5-propyl 2-thiouracil; RV, right ventricular; SF, shortening fraction; Tmod, tropomodulin; TOT, Tmod overexpressing transgenic. 
tive termination of polymerization. Tropomodulin (Tmod) is a component of the thin filament complex in cardiac muscle where, as in skeletal muscle, the protein colocalizes where pointed ends of actin filaments are located $(10,11)$. Tmod/actin filament stoichiometry appears critical for maintaining actin filament structure (12-14). Overexpression of Tmod in cultured primary cardiomyocytes leads to myofibril degeneration, consistent with a role for Tmod in the maintenance of myofibril organization and for the necessity of regulated Tmod expression (15). Collectively, these in vitro studies suggest that cardiac-specific overexpression of Tmod in transgenic mice would be a useful experimental model system to study cardiomyopathy caused by myofibril degeneration.

Tmod overexpressing transgenic (TOT) mice were created with the expectation that Tmod overexpression would cause thin filament breakdown leading to myofibril degeneration and loss of systolic function. Indeed, TOT mice suffer from dilated cardiomyopathy which resembles human disease with respect to variation in both the type and severity of pathological changes. The timing of TOT cardiomyopathy results from activation of the transgene in the ventricle which occurs within $2 \mathrm{wk}$ after birth (16). This report describes TOT mice showing the most severe phenotype: a dilated cardiomyopathy occurring 3-4 wk after birth accompanied by symptoms of heart failure.

\section{Methods}

Construction of the $\alpha$-myosin heavy chain (MHC)-Tmod transgene. The $\alpha-5.5$ wild-type promoter has been described previously (17) and has been tested in vivo (18). Full-length mouse Tmod cDNA (19) was inserted downstream from the $\alpha$-MHC promoter in a unique SalI restriction site of the plasmid (clone 26, provided by J. Robbins, The Children's Hospital and Research Foundation).

Production of transgenic mice and transgene dosage determination. DNA constructs were prepared for injection as described previously (18). Briefly, plasmid containing the $\alpha$-MHC promoter/Tmod construct was digested with NotI to release the transgene DNA fragment which was separated from vector by agarose gel electrophoresis. The fragment was purified using QIAquik (QIAGEN, Chatsworth, CA), phenol/chloroform extracted, alcohol precipitated, and resuspended for injection. Genomic DNA was isolated from tail clips digested at $55^{\circ} \mathrm{C}$ overnight with $100 \mathrm{mM}$ Tris- $\mathrm{HCl}, \mathrm{pH} 8.5,5 \mathrm{mM}$ EDTA, $200 \mathrm{mM} \mathrm{NaCl}, 0.2 \%$ SDS, and $5 \mu \mathrm{g} / \mathrm{ml}$ proteinase $\mathrm{K}$ (Boehringer Mannheim, Indianapolis, IN). DNA was purified from the digest using Phase Lock Gel I (Heavy; 5 Prime-3 Prime, Boulder, CO) and resuspended in $1.5 \mathrm{ml}$ of $\mathrm{dH}_{2} \mathrm{O}$. A sample of genomic DNA sample $(30 \mu \mathrm{l})$ was digested with EcoRI, precipitated, resuspended, and loaded for slot blot analysis at a concentration of $5 \mu \mathrm{g}$ per slot (The Convertible Filtration Manifold System, 48 wells; GIBCO BRL, Gaithersburg, MD). Blotted DNA samples were analyzed using full-length Tmod riboprobe (19) and glyceraldehyde 3-phosphate dehydrogenase (GAPDH) riboprobe (pTRI-GAPDH-mouse; Ambion, Inc., Austin, TX). Genomic copy number was standardized relative to the GAPDH signal to correct for variation in sample loading. Three different founder lines $(65,14$, and 72$)$ showed either one or two copies of the transgene. Mating of a founder line (65) transgenic female (two transgene copies) and nontransgenic male produced heterozygous $\mathrm{F}_{1}$ offspring. These $F_{1}$ siblings were then mated to produce $F_{2}$ mice with litter ratios of 1 normal (no transgene): 2 heterozygotes ( 2 copies of the transgene): 1 homozygote ( 4 copies of the transgene). The $F_{2}$ heterozygotes showed elevated Tmod expression without juvenile morbidity or mortality (data not shown) and are the subject of ongoing study. The $\mathrm{F}_{2}$ homozygotes showed a severe cardiomyopathic phenotype and are referred to as TOT mice. The correlation between gene dosage and phenotype of the founder line in this study (65) was also seen in alter- nate founder lines (14 and 72) as well as in cross-breeding between founder lines (data not shown). TOT mice were derived from line 65 .

Determination of $m R N A$ expression levels. Approximately $2 \mu \mathrm{g}$ of total RNA isolated from freshly excised hearts using TRI reagent (Molecular Research Center, Inc., Cincinnati, OH) was loaded for slot blot analysis (The Convertible Filtration Manifold System). Antisense oligonucleotide probes identical to those used in Jones et al. (20) were labeled with $\gamma^{-32} \mathrm{P}$ (DuPont/New England Nuclear, Boston, MA) using T4 polynucleotide kinase (GIBCO BRL). Blots were hybridized for $4 \mathrm{~h}$ to labeled probes at $55^{\circ} \mathrm{C}$, washed three times with $1 \times \mathrm{SSC}, 0.5 \% \mathrm{SDS}$, and then placed on an imaging screen for overnight exposure. Counts absorbed by the screen were quantitated using a PhosphorImager (Molecular Dynamics, Sunnyvale, CA).

Preparation of samples for microscopic analyses. After $\mathrm{CO}_{2}$ asphyxiation, hearts were immediately removed and prepared for fixation by one of the following: (a) blood was removed by flushing with relaxing buffer ( $5 \%$ dextrose, $25 \mathrm{mM} \mathrm{KCl}$ in PBS) administered by gravity flow through a needle inserted into the apex of the heart; $(b)$ the heart was placed in relaxing buffer and blood was expelled from the chambers by gently applying pressure to the heart walls; or $(c)$ the heart was placed directly into fixative. Hearts were fixed in Histoprep formalin (HC-200; Fisher Scientific, Pittsburgh, PA) for histological analyses. Serial sections were cut on a Zeiss Microm HM 325 at $5 \mu \mathrm{m}$ thickness and stained with hematoxylin (Fisher Scientific) and eosin (Sigma Chemical Co., St. Louis, MO). The pulmonary system in Fig. 3 was fixed in situ by perfusion with $4 \%$ paraformaldehyde in PBS and the combined heart/lung tree was processed for paraffin embedding, sectioning, and microscopic analysis.

For fluorescent antibody staining, sections were deparaffinized in Hemo-D (Fisher Scientific Inc.) and rehydrated through a series of graded alcohols to distilled water. To enhance antibody reactivity with tropomyosin and Tmod, sections were lightly digested for $5 \mathrm{~min}$ at room temperature in $0.1 \mathrm{mg} / \mathrm{ml} \mathrm{CaCl}_{2}(\mathrm{pH} 7.7)$ containing $0.1 \mathrm{mg} / \mathrm{ml}$ trypsin (1:250; Difco Laboratories Inc., Detroit, MI). Fluorescence staining was carried out as described previously (21). Sections were labeled with antibodies to Tmod (described in reference 22), tropomyosin (T-9283; Sigma Chemical Co.), desmin (213M; Biomeda, Foster City, CA), and actin (clone C4, kindly provided by J. Lessard, The Children's Research Foundation). Primary antibodies were detected by fluorescently tagged secondary goat anti-mouse IgG (Cy 5; Jackson ImmunoResearch Laboratories, Inc., West Grove, PA) and goat anti-rabbit IgG (FITC, F-0382; Sigma Chemical Co.). Sections were mounted in Vectashield medium (Vector Laboratories, Inc., Burlingame, CA) for viewing by confocal microscopy.

Single cardiomyocytes were prepared for fluorescence labeling and viewed by confocal microscopy as described previously (23).

Light microscopy. High magnification images were created by photographing sections onto 35-mm Ektachrome T160 color slide film using an Olympus BH-2 RFCA microscope equipped with a Nikon VFX-11A light meter, Nikon FX-35DX camera, didymium filter, and a $\times 40$ oil immersion objective. $35-\mathrm{mm}$ slides were converted into digitized images using the Nikon LF-3500 slide scanner (Nikon Inc., Melville, NY). Low magnification sections were scanned directly from the microscope slide. Digital images were printed using a Codonics NP-1600 dye sublimation printer (Codonics, Akron, OH).

Confocal microscopy and image analysis. Labeled sections or cells were viewed using a confocal microscope (CLSM 2010; Molecular Dynamics) capable of laser excitation by 488,568 , and/or $647 \mathrm{~nm}$ wavelengths with various objectives. Image analysis was performed using Imagespace software (version 3.2) on a Silicon Graphics Indy platform (Silicon Graphics, Mountain View, CA). A $512 \times 512$ pixel area $(0.21 \mu \mathrm{m})$ was scanned as a stack of single image slices along the vertical $(Z)$ axis of the cell. Data sets were processed using threedimensional mean pixel intensity filter to smooth the image, and all micrographs are a single slice of the image stack chosen from the central region of the cell. Processed images were printed using a Codonics NP-1600 dye sublimation printer.

Electron microscopy. Samples were prepared and viewed as de- 
scribed previously (24). Sarcomere length was calculated from a minimum of 5 sarcomeres using three sections from two different control and TOT mice for a total of 25 control and 25 TOT sarcomeres. Significance was determined using Student's $t$ test.

Determination of Tmod protein expression. Equivalent amounts of total protein were separated by SDS-PAGE on a $10 \%$ gel and transferred to nitrocellulose. The region corresponding to apparent mobility of Tmod was excised from the blot and labeled with anti-Tmod antibody. Bound antibody was detected by labeling with a Vistra ECF Western blot kit (Amersham Corp., Arlington Heights, IL) as directed by the manufacturer. Signal intensity was quantitated using a Storm 860 fluorimager (Molecular Dynamics) and calculated as the product of average pixel intensity in the band multiplied by the area of the band.

Echocardiography. After sedation with isofluorane inhalation and shaving the anterior chest area, mice were placed on a thermally controlled foam pad. Temperature monitored via a rectal probe was maintained between 35 and $38^{\circ} \mathrm{C}$ using a warming pad and heat lamps. Mice were allowed to breathe spontaneously. Echocardiography was performed using a Sonos 2500 Ultrasound System equipped with a 7.5-MHz transducer (Hewlett-Packard Co., Andover, MA). To place the heart in the midfield of the ultrasound sector for optimal axial resolution, the transducer was fashioned with a latex balloon filled with warm acoustic gel to create a $1-\mathrm{cm}$ standoff between the chest wall and transducer face with a depth setting of the system at 3 or $4 \mathrm{~cm}$. A warm acoustic gel layer was applied to the left hemithorax and the probe was placed on the chest avoiding excessive pressure. Twodimensionally guided M-mode echocardiography of left ventricle and aortic annulus short axes were recorded on S-VHS video and a strip chart at $100 \mathrm{~mm} / \mathrm{s}$. Pulsed wave Doppler interrogation was performed of the ascending aorta from a superior angulation. LV end-diastolic and end-systolic dimension and thickness (LVED, LVES, $\mathrm{h}_{\mathrm{d}}, \mathrm{h}_{\mathrm{s}}$, respectively), end-diastolic septal thickness $\left(s_{d}\right)$, ejection time (ET), and the RR interval were measured from the M-mode echocardiogram. End-diastole was defined as the largest LV dimension; end-systole was defined as the smallest LV dimension. Aortic root diameter (Ao) was also measured from the M-mode echocardiogram. Blood pressure $(\mathrm{P})$ was obtained by a cuff tail measurement. The aortic time velocity integral (AoTVI) was measured by on-line tracing the Doppler spectral profile of the ascending aortic velocity. LV mass was calculated as LV mass $(\mathrm{mg})=[0.8][1.04]\left[\left(L V E D+h_{\mathrm{d}}+s_{\mathrm{d}}\right)^{3}-\right.$ $\left.(L V E D)^{3}\right][1000]$. LV performance was assessed by calculating shortening fraction $(S F=[L V E D-L V E S] / L V E D)$, heart rate-corrected velocity of circumferential fiber shortening $\left(V C F=[S F]\left[R R^{0.5}\right] /\right.$ $[E T])$, and cardiac output $\left(\mathrm{CO}=[\mathrm{Pi}][\mathrm{Ao} / 2]^{2}[\mathrm{AoTVI}]\right)$. All data are shown as mean \pm SD. Statistical analyses were performed using SAS software (Carey, NC). Paired Student's $t$ tests were used to determine differences in variables in the same mouse between conditions. Unpaired Student's $t$ tests were used to determine differences in variables between mice. Values were considered significant at $P<0.05$.

Isolated heart physiology. The working heart preparations were performed as described previously (25).

\section{Results}

Cardiomyopathic changes in TOT mouse hearts. TOT mice with severe cardiomyopathy were readily identified 10-21 d after birth by their decreased physical stamina compared with healthy littermates, which became apparent during the course of routine maintenance. These TOT mice also exhibited proptosis with unusually dark eyes. Approximately $70 \%$ of TOT mice showing marked external signs died within 2-3 d, but TOT mice always died if overstressed with physical handling for examination, tissue sampling, or cage maintenance. Using these external signs as a guide, affected TOT mice were identified and killed for assessment of cardiac structure. Several pathological changes were apparent in gross examination of transgenic hearts, including global dilation with marked right side atrial and ventricular enlargement (Fig. 1). The translucent appearance of the right ventricular (RV) myocardial wall indicated significantly decreased thickness relative to chamber size (Fig. $1 A$ ), which was confirmed upon viewing the bisected heart (Fig. 1, $B$ and $C$ ). Sizes of all four chambers were markedly increased and thrombi were often present within the left atrium. The normal trabecular pattern was maintained in the right atrium, even in severe cases of enlargement.

A trend toward lower body weight was found in TOTs $(6.8 \pm 0.9 \mathrm{~g})$ compared with controls $(8.4 \pm 1.26 \mathrm{~g})$ which was not statistically significant $(P>0.02)$ in the groups tested (control $n=7$, transgenic $n=6)$. In contrast, the difference in heart weight between TOTs $(92.7 \pm 7.0 \mathrm{mg})$ and controls $(46.1 \pm 5.0 \mathrm{mg})$ was highly significant $(P<0.00001)$. However, lung weights were comparable between TOTs $(80.8 \pm 22.3 \mathrm{mg})$ and controls $(80.3 \pm 9.6 \mathrm{mg})$, consistent with absence of pathological changes in lung sections (see below). These changes are reflected in heart/body weight ratios of $5.5 \mathrm{vs} .13 .5 \mathrm{mg} / \mathrm{g}$ compared with lung/body weight ratios of $8.6 \mathrm{vs} .11 .9 \mathrm{mg} / \mathrm{g}$ for controls and TOTs, respectively.

TOT and nontransgenic control hearts were processed for histological analysis (Fig. 2). Sections of hearts comparable in size were taken from a 3.5-mo-old control mouse and 3.5wk-old TOT mouse. Comparison of these sections (Fig. $2 \mathrm{~A}$ ) showed several pathological changes typically observed in TOT hearts, such as dilation of all chambers with a marked increase in the size of the right ventricle $(r v)$, thinning of ventricular walls and the septum in relation to chamber area, and presence of a thrombus in the left atrium (Fig. $2 \mathrm{~A}$, arrow). Thinning of ventricular walls indicates that the significant enlargement of the heart was not accompanied by a hypertrophic response. Lack of cardiac hypertrophy was also apparent when hearts from age-matched mice were compared (Fig. $2 \mathrm{~B}$ ). Aside from areas in left atrial walls located next to a thrombus, there was no increase in mononuclear cell infiltrate throughout the myocardium. Examination of TOT mouse ventricular walls at higher magnification showed karyomegalic nuclei. The percentage of cell volume occupied by the nucleus in TOT hearts was significantly greater than in controls (Fig. 2, $C$ and $D$, $22.1 \pm 2.6$ vs. $12.4 \pm 2.5 \% ; P<0.00001)$. Nuclear counts of both control and TOT mouse sections showed no increase in nuclear number, indicating no increase in cellular proliferation. Staining intensity shows more heterogeneity in fibers from TOT mice, and fibers appear thinner and less compact in TOT hearts. Sections stained with Gomori trichrome to detect fibrosis showed no difference between nontransgenic controls and TOT mice (data not shown). Lungs appeared normal, with no pathological changes suggestive of pulmonary edema such as mononuclear infiltrate or fluid accumulation in the alveolar bed (data not shown).

Tmod immunoreactivity in samples of nontransgenic hearts was barely detectable (Fig. $3 \mathrm{~A}$ ). In comparison, immunoreactivity in TOT mouse hearts scanned at identical settings was intense and widespread throughout both atrial and ventricular walls (Fig. 3, $B$ and $C$ ). Pulmonary vasculature was also labeled by anti-Tmod antibody in TOT samples (Fig. $3 C$, arrows), consistent with the distribution of $\alpha$-MHC promoter activity (26).

TOT mice show loss of myofibrillar organization. Confocal microscopy was used for visualization of sarcomeric organi- 

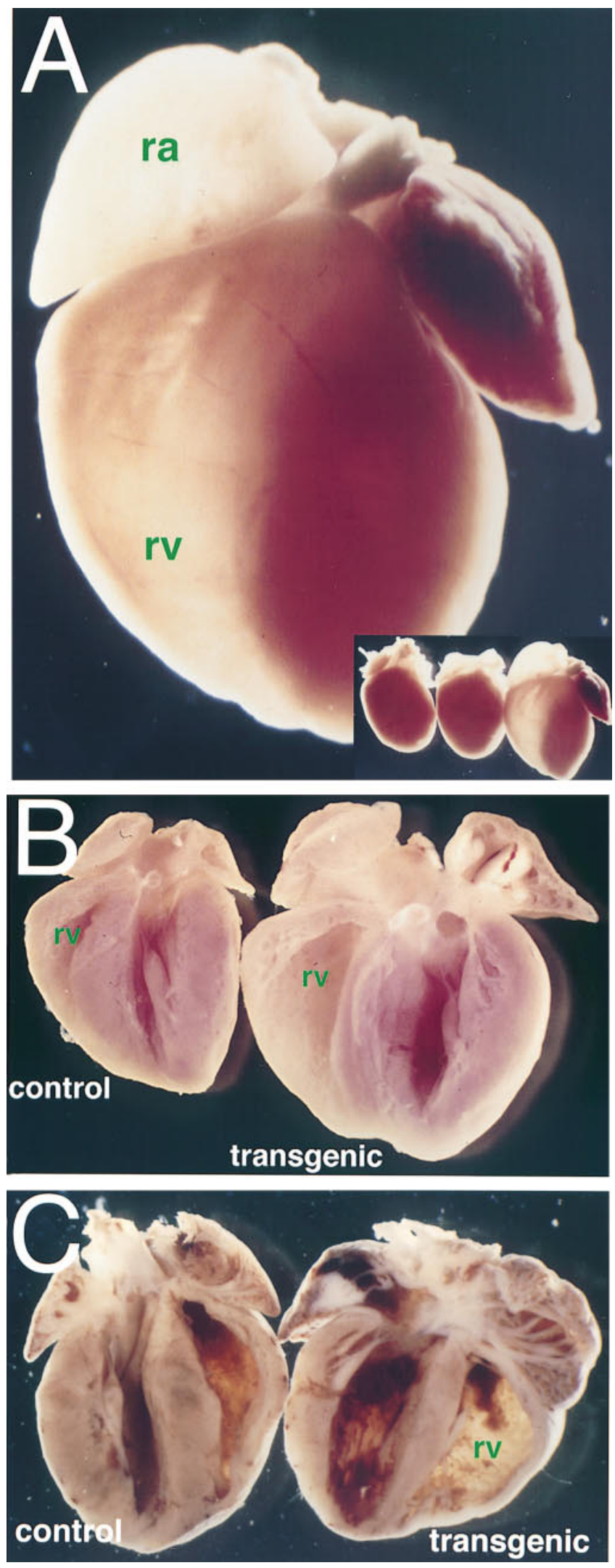

Figure 1. Pathological changes in TOT hearts consistent with dilated cardiomyopathy. Views of hearts from TOT and nontransgenic control mice. The most prominent feature in a TOT heart $(A)$ is marked enlargement of the right atrium $(r a)$ and right ventricle $(r v)$. The right ventricle is translucent due to thinning of the wall, and the heart is
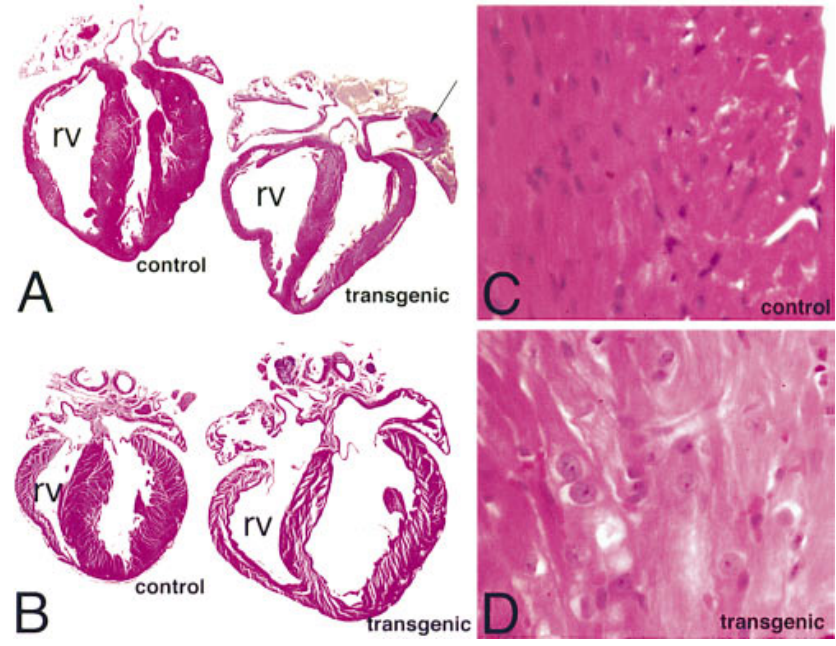

Figure 2. Histological analysis of myocardial tissue from TOT hearts. Sections of hearts from nontransgenic control and TOT mice stained with hematoxylin and eosin. Sections of size-matched hearts $(A)$ from a 14-wk-old control mouse and a 3.5-wk-old TOT mouse. Dilation of the TOT heart is evident particularly in the RV chamber $(r v)$. Although comparable in size, transgenic chamber walls are markedly thinner and atria are abnormally large. A thrombus is often found in the left atrium of transgenic animals (arrow). Sections of agematched hearts $(B)$ from control and transgenic mice shown 3 wk after birth. Dilation of the transgenic heart is evident, but a hypertrophic increase in chamber wall thickness is absent. High magnification views of the LV wall in control $(C)$ and TOT mice $(D)$. Enlarged nuclei are evident in the transgenic sections $(D)$, but the number of nuclei per field is similar between control and TOT hearts. $A$ and $B$, $\times 6 ; C-F, \times 200$.

zation in heart sections of TOT and nontransgenic control mice labeled with antibodies to Tmod and actin (Fig. 4, top), Tmod and tropomyosin (Fig. 4, middle), and actin and desmin (Fig. 4, bottom). Normal myocardium showed a striated pattern of fluorescence with all antibodies used, but loss of myofibril organization and sarcomeric dysgenesis were apparent in sections of myocardium from TOT mice. Typical findings of TOT mouse cardiac myofibril condition included loss of parallel alignment, heterogeneity of staining intensity, and curviform appearance (Fig. 4, middle).

The relationship between Tmod and $\alpha$-actinin distribution was observed in isolated cardiomyocytes from TOT and control mice. Normal cardiomyocytes contain a dense packed parallel aligned array of myofibrils which show alternating bands of Tmod and $\alpha$-actinin reactivity (Fig. 5, left). In cardiomyocytes isolated from TOT hearts, the normal alternating pattern

significantly larger than control hearts from nontransgenic siblings ( $A$, inset, TOT heart on right with two nontransgenic hearts). Agematched hearts $(B)$ from control and TOT mice are bisected (without prior fixation) to show significant increases in chamber sizes throughout the heart, particularly dilation of the right ventricle. Size-matched hearts $(C)$ from a 14 -wk-old control mouse and a 3.5 -wk-old TOT mouse are bisected (after formalin fixation) to show overall thinning of myocardium, dilation of chambers, and thrombus formation in the left atrium. 

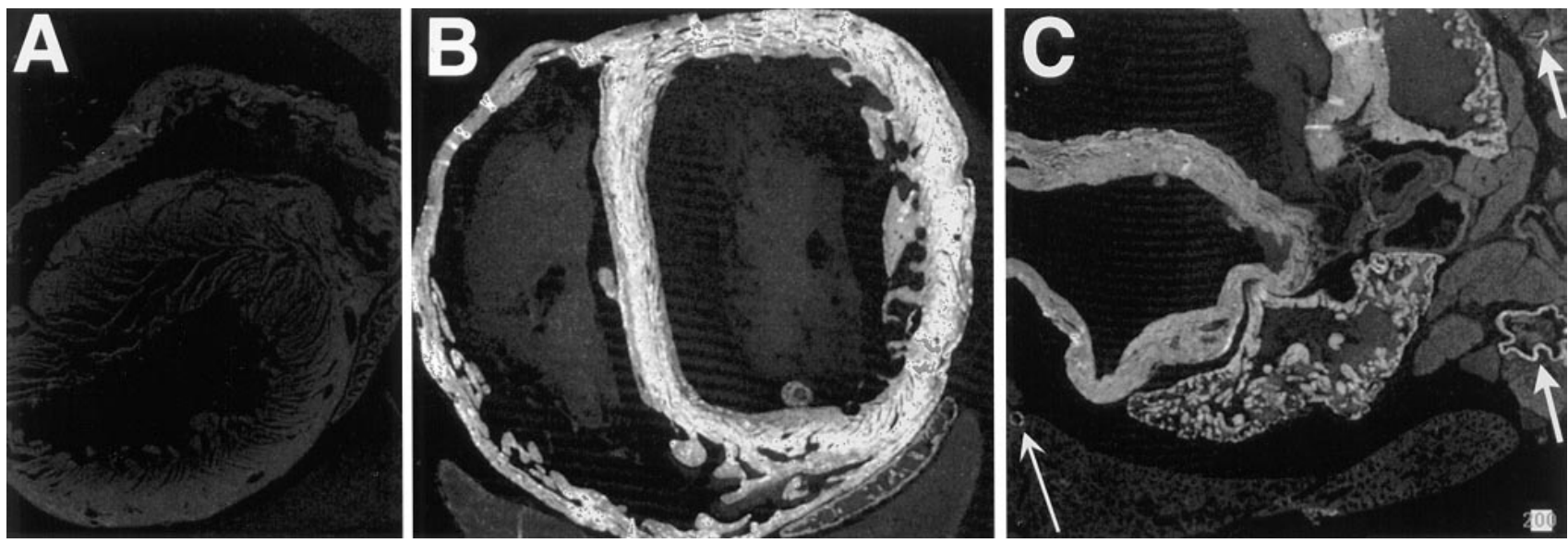

Figure 3. Tmod distribution in TOT hearts. Sections of heart samples from nontransgenic control $(A)$ and TOT $(B$ and $C)$ mice show increased Tmod immunoreactivity throughout the myocardium. All images were scanned and processed identically to permit subjective comparison of labeling intensities between the control and TOT samples. Tmod immunoreactivity is substantially increased throughout the ventricular walls of TOT mice $(B$ and $C$ ). Tmod protein also accumulates in the atria and walls of the pulmonary myocardium (arrows). Bar, $200 \mu \mathrm{m}$.

\section{control}
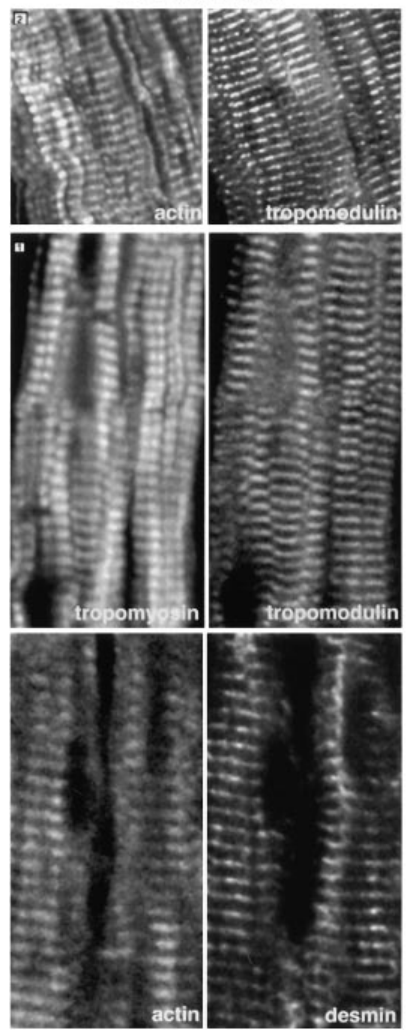

\section{transgenic}
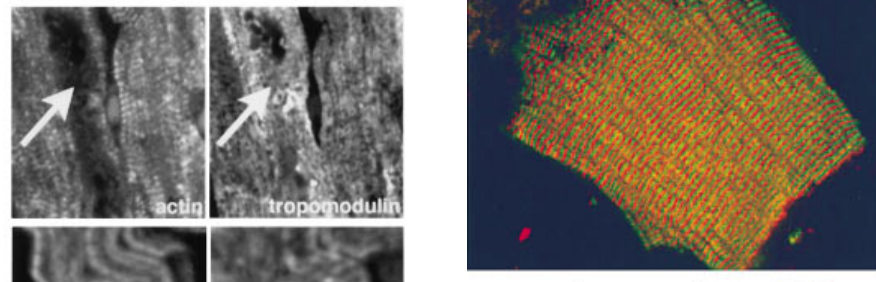

nontransgenic control

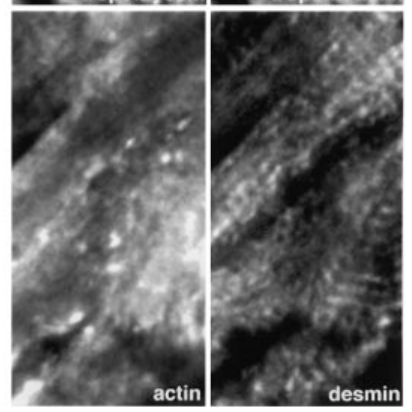

Figure 4. Disruption of myofibril organization in TOT hearts. Sections of heart tissue from nontransgenic control (left) and TOT (right) mice labeled with antibodies as designated in the lower right corner of each photo. Control sections possess a well organized myofibrillar array with clearly defined cross-striations. In comparison, TOT sections show widespread Tmod immunoreactivity and poorly organized sarcomeric actin. TOT sections show thread-like myofibrils labeled with antiactin antibody (top) or antitropomyosin antibody (middle) but myofibril degeneration is most apparent in a region of increased Tmod immunoreactivity (top, arrows). Sections with almost total loss of discernible sarcomeric actin still show desmin striations. Bar at top left, $2 \mu \mathrm{m}$; middle left, $1 \mu \mathrm{m}$ (same scale for bottom).

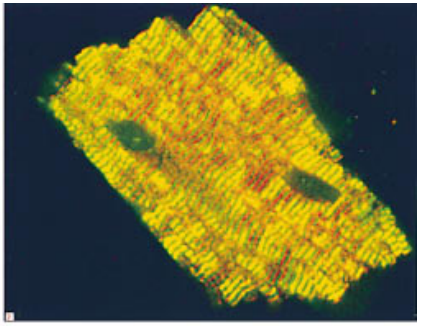

transgenic overexpression
Figure 5. Single cardiomyocytes show changes in contractile protein organization. Localization of Tmod (green) and $\alpha$-actinin (red) in cardiomyocytes isolated from a nontransgenic control and TOT mouse. Control cells show little or no overlap (yellow) between Tmod and $\alpha$-actinin labels in control cells, but the abundant yellow signal in transgenic cells indicates aberrant colocalization of Tmod with $\alpha$-actinin. Tmod staining in TOTs often appears patchy, indicating that accumulation of Tmod protein is not uniform throughout the cell interior. Bar, $2 \mu \mathrm{m}$.

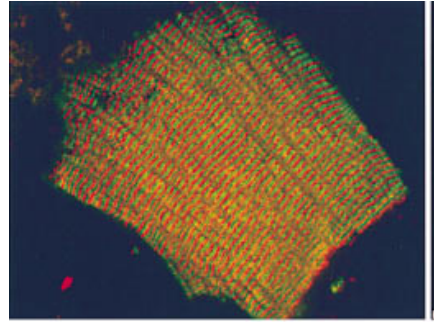

of Tmod and $\alpha$-actinin immunoreactivity was disrupted and Tmod was abnormally coincident with $\alpha$-actinin (Fig. 5, right). Three-dimensional image analysis showed that Tmod accumulated in patches rather than uniformly throughout the cardiomyocyte. $^{2}$

Sections from TOT hearts and nontransgenic controls were viewed by electron microscopy to observe sarcomeric ultrastructure. Normal myofibrils showed typical parallel alignment with well-organized $\mathrm{Z}, \mathrm{A}$, and $\mathrm{M}$ bands (Fig. 6, $A$ and $B$, arrowheads, arrow, and asterisk, respectively). In comparison, myofibrils in TOT hearts show lack of organization and loss of parallel arrangement (Fig. 6, $C-F$ ). Myofibrils were absent

2. Animated movies of reconstructed cardiomyocytes from TOT and control mice which show differences in Tmod distribution are available for viewing in the "Movies" section of our World Wide Web site at: http://heart.chmcc.org 

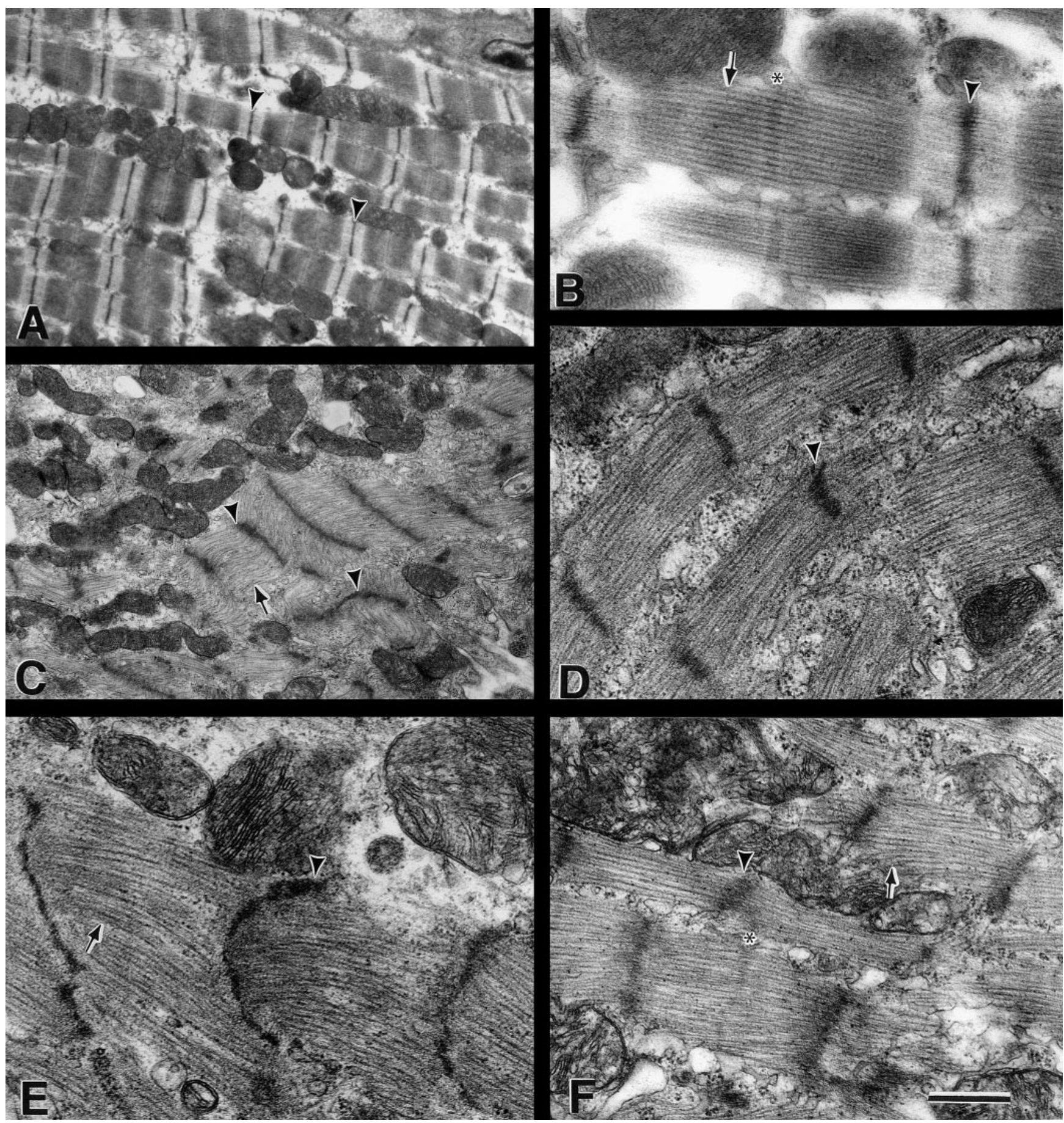

Figure 6. Representative transmission electron micrographs from the hearts of control and TOT mice. All samples were obtained from the left ventricle except for $F$, which was taken from the right ventricle. Myofibrils (arrows) in controls $(A$ and $B)$ were arranged in well-organized parallel arrays while those in TOTs were poorly organized and frequently curved within a single sarcomere. In comparison to the compact $\mathrm{Z}$ bands (arrowheads) characteristic of control myofibrils, $\mathrm{Z}$ bands in TOTs $(C-F)$ were frequently curved and of varying width and thickness in a single myofibril. M bands (asterisks) in TOTs were not found in the left ventricles and rarely found in the right ventricles. Mitochondria in controls were usually found in limited areas between the parallel arrays of myofibrils, while in TOTs large areas of the cardiomyocytes lacked myofibrils and were filled with numerous mitochondria ( $C$, upper left). Scale bar, $2.7 \mu \mathrm{m}$ for $A ; 0.5 \mu \mathrm{m}$ for $B, D$, and $E ; 1.8 \mu \mathrm{m}$ for $C$; and $0.7 \mu \mathrm{m}$ for $F$.

from large areas of the cell and numerous mitochondria were present (Fig. $6 \mathrm{C}$ ), consistent with ultrastructural characteristics of degenerating cardiomyocytes (27). Actin and myosin filaments were found without discernible $\mathrm{Z}$ bands in some areas, whereas in other regions distinct closely spaced sarcomeres were seen. Myofibrils were curved with distorted $\mathrm{Z}$ bands as observed by confocal microscopy (see Figs. 4 and 10).

Sarcomeric length in micrographs was determined by mea- 


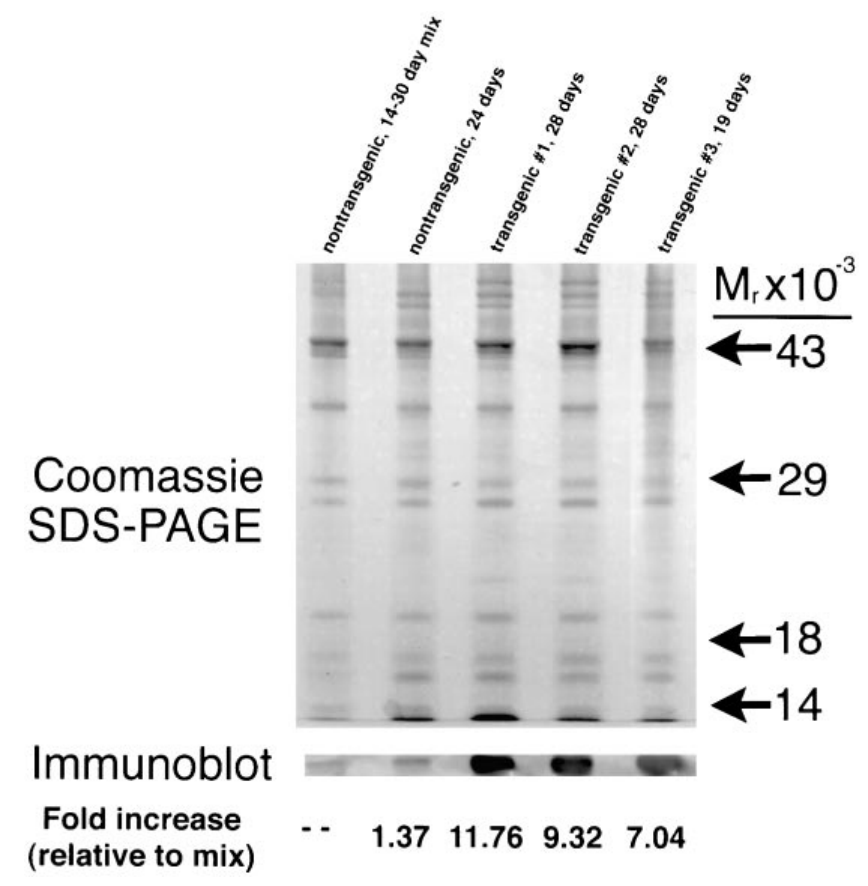

Figure 7. Tmod protein expression is substantially increased in TOT hearts. Coomassie SDS-PAGE and the immunoblot showing the increased level of Tmod protein in TOT hearts. Equivalent amounts of cardiac protein lysates were loaded and subjected to overnight electrophoresis followed by either staining with Coomassie brilliant blue dye or transfer to nitrocellulose. The immunoblot region corresponding to the $M_{\mathrm{r}}$ of Tmod $(\sim 43 \mathrm{kD})$ was excised and labeled with antiTmod antibody. Bound antibody was detected by enhanced chemifluorescence and signal was quantitated by fluorimager analysis (see Methods). Samples loaded in each lane are indicated across the top of the gel. Pooled heart tissue from nontransgenic controls was used as a reference to minimize variations in Tmod level between individual animals. In comparison to this pooled sample, a nontransgenic animal has a similar Tmod level, whereas samples from various TOT mice show increases ranging from 7- to almost 12 -fold. Mobility $\left(M_{\mathrm{r}} \times\right.$ $10^{-3}$ ) of molecular weight standards is shown at the right of the gel.

surement from the inside edge of one $\mathrm{Z}$ band to the outside edge of the next nearest band. Control nontransgenic sarcomeres averaged 2.02 $\pm 0.172 \mu \mathrm{m}$ (range $=1.83-2.41 \mu \mathrm{m}$, standard error $=0.034$ ), as expected for normal sarcomeres. In areas of TOT sections where $\mathrm{Z}$ bands could be distinguished, average sarcomere length was $1.08 \pm 0.157 \mu \mathrm{m}$ (range $=0.83-$ $1.33 \mathrm{~mm}$, standard error $=0.031$ ). Thus, sarcomeric length in TOTs is $53 \%$ shorter than that of control mice processed and imaged at the same time.

Tmod protein expression level. Tmod protein expression was determined relative to an average Tmod expression level based upon a cardiac lysate prepared from a mixture of nontransgenic control hearts varying in age from 14-30 d. Comparable Tmod expression was present between the control mixture (Fig. 7, first lane) and a 24-d-old mouse sample (Fig. 7, second lane). Two different TOT mice at $28 \mathrm{~d}$ after birth showed substantially increased Tmod expression over 9-11-fold in the two samples tested (Fig. 7, third and fourth lanes). A third sample from a younger TOT mouse at $19 \mathrm{~d}$ (Fig. 7, fifth lane) also showed an increase in Tmod expression at sevenfold over nontransgenic levels.
Expression of genes associated with cardiomyopathy. Expression level of mRNAs for genes involved in cardiac failure and/or hypertrophic responses in TOT mice were compared with nontransgenic controls (Fig. 8). Differences in loading between samples were corrected relative to GAPDH. Since myosin light chain (ventricular isoform) (MLC-2v) expression levels were comparable between control and TOT mice and were unaffected by cardiomyopathic changes in other experimentally induced cardiomyopathies $(20,28)$, MLC-2v was used as a reference to calculate relative changes in mRNA expression. SERCA mRNA showed a significant drop in expression level (42\% compared with control, $P=0.00002$ ), whereas significantly increased mRNA expression was found for $\beta$-MHC $(180 \% ; P=0.00003)$ and atrial natriuretic factor $(157 \% ; P=$ 0.0002). Other mRNAs which did not show a significant change $(P>0.01)$ in expression level included $\alpha$-MHC, skeletal actin, cardiac troponin $\mathrm{C}$, and titin.

Echocardiography shows severe dilated cardiomyopathy. Findings of LV dilatation, thinning, and poor systolic performance were clearly demonstrated by M-mode echocardiography (Fig. 9). Hemodynamic assessment of echocardiographic results using 3-4-wk-old mice (Table I) demonstrated extreme LV dilatation (increased LVED) and thinning of the LV posterior wall. LV dilatation in TOT mice was so severe that indexed LV mass was significantly greater (despite thinner myocardium) than in control mice. LV systolic performance was also severely depressed in TOT mice as reflected by decreased SF, velocity of circumferential fiber shortening, and cardiac output.

Altered contractile properties of TOT hearts. Cardiac function of hearts from size- and sex-matched TOT mice and nontransgenic controls was assessed using isolated Langendorff (retrograde perfusion, nonworking) preparations. TOT mouse hearts differed from controls in both their contractile and relaxing properties under identical levels of retrograde flow.

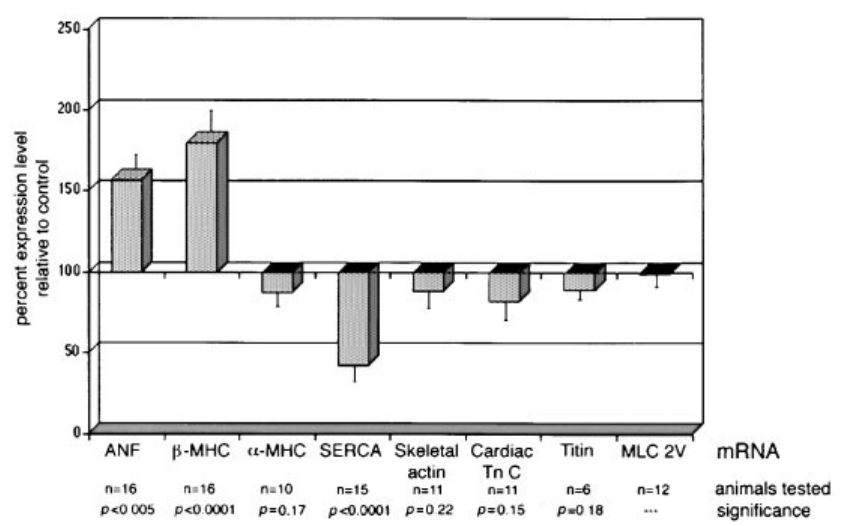

Figure 8. Comparison of mRNA expression levels in hearts of TOT mice. Analyses of cardiac gene expression in TOT mice for genes which may be involved in hypertrophic/failure responses. Results are shown as percent expression for TOT mRNAs relative to nontransgenic levels. Equivalent expression between controls and TOTs is presented as $100 \%$ (baseline) with values above and below $100 \%$ representing increased and decreased expression and values, respectively. Significance values were calculated relative to MLC-2v. Error bars represent standard errors of the mean. All hybridization signals were normalized for variations in loading with respect to GAPDH. 


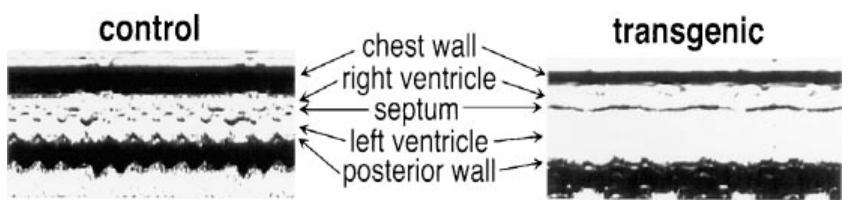

Figure 9. Echocardiography shows cardiomyopathic changes. Representative M-mode echocardiograms from control and TOT mice. The TOT mouse shows a much greater LV cavity dimension and much thinner posterior wall. Note flattening of both the septal and LV posterior wall motion in the TOT compared with vigorous motion in the control mouse.

TOT hearts produced maximal rates of pressure development which were significantly reduced relative to the control hearts: $+d P / d t$ was reduced by $59 \%$ and $-d P / d t$ was reduced by $52 \%$ (Table II). The decreased $+d P / d t$ indicates that TOT hearts have significantly reduced contractility (longer time to develop peak ventricular pressure), while the decreased $-d P / d t$ shows that relaxation is also prolonged. Heart rate was also $11 \%$ lower than normal in TOT hearts $(P \leq 0.0001)$.

Treatment of TOT mice with 5-propyl 2-thiouracil (PTU) inhibits cardiomyopathy. The $\alpha$-MHC promoter of the transgene in TOT mice is responsive to thyroid hormone levels (29). Experimentally induced hypothyroidism by diet supplementation with PTU inhibits $\alpha$-MHC promoter driven transgene expression for chloramphenicol acetyltransferase (18) and $\beta$-tropomyosin (30). If cardiomyopathy in TOT mice results from Tmod overexpression, then PTU supplementation should prevent development of Tmod-mediated heart disease. Throughout a 6-wk period of PTU diet supplementation, TOT mice identified by genomic Southern blot analyses showed no external signs of cardiomyopathy. As expected, TOT mice developed external signs of cardiomyopathy after withdrawal of PTU. External symptoms of cardiomyopathy quickly appeared within $4 \mathrm{~d}$, a rapid progression of cardiomyopathy relative to the 2-4-wk time course required to develop similar symptoms

Table I. Hemodynamic Assessment by Echocardiography

\begin{tabular}{lccc}
\hline \multicolumn{1}{c}{ Hemodynamic index } & Control & Transgenic & $P$ value \\
\hline & $n=3$ & $n=4$ & \\
Weight (g) & $13 \pm 5$ & $9 \pm 3$ & NS \\
Heart rate (bpm) & $504 \pm 16$ & $372 \pm 78$ & 0.046 \\
RV end-diastolic dimension (mm) & $1.3 \pm 0.4$ & $1.6 \pm 0.1$ & NS \\
LVED (mm) & $2.9 \pm 0.2$ & $4.4 \pm 0.6$ & 0.01 \\
LV wall thickness, diastole (mm) & $0.7 \pm 0.3$ & $0.4 \pm 0.2$ & NS \\
LV wall thickness, systole (mm) & $1.3 \pm 0.2$ & $0.7 \pm 0.2$ & 0.008 \\
Intraventr. septal thickness (mm) & $0.7 \pm 0.3$ & $0.7 \pm 0.0$ & NS \\
LV mass (mg) & $47 \pm 12$ & $68 \pm 27$ & NS \\
LV mass/body weight (mg/g) & $3.7 \pm 0.9$ & $7.6 \pm 0.4$ & 0.0007 \\
Ao (mm) & $1.0 \pm 0.2$ & $0.9 \pm 0.08$ & 0.07 \\
Shortening fraction (\%) & $55 \pm 10$ & $15 \pm 6$ & 0.001 \\
Velocity-fiber shortening (circ/s) & $3.5 \pm 1$ & $0.8 \pm 0.3$ & 0.004 \\
LV stroke volume (mcl/beat) & $24 \pm 12$ & $6 \pm 3$ & NS \\
LV cardiac output (mcl/min) & $12000 \pm 5000$ & $2400 \pm 1000$ & NS \\
LV card output/weight (mcl/min/g) & $750 \pm 194$ & $318 \pm 242$ & 0.098 \\
LV wall stress (g/cm $\left.{ }^{2}\right)$ & $15, n=2$ & $275, n=1$ & \\
& & &
\end{tabular}

Table II. Means \pm SEM of Measured Cardiac Parameters

\begin{tabular}{lcll}
\hline & Control & + T mod & $\%$ Change \\
\hline Langendorff heart & $n=4,40$ & $n=4,40$ & \\
Heart rate $(\mathrm{bpm})$ & $329 \pm 5$ & $292 \pm 6^{*}$ & $-11 \%$ \\
$+d P / d t(\mathrm{mmHg} / \mathrm{ms})$ & $1479 \pm 147$ & $603 \pm 31^{*}$ & $-59 \%$ \\
$-d P / d t(\mathrm{mmHg} / \mathrm{ms})$ & $891 \pm 89$ & $426 \pm 23^{*}$ & $-52 \%$ \\
\hline
\end{tabular}

$* P \leq 0.0001$, transgenic vs. control, unpaired Student's $t$ test.

in euthyroid TOTs. Hearts were harvested from TOT mice after PTU supplementation had been discontinued and external symptoms of cardiomyopathy were noted. For comparison, control hearts were harvested from nontransgenic littermates which were maintained with the same PTU-supplemented diet. Histological analyses of these hearts showed a trend toward dilation (Fig. 10, sides), but the increase in overall heart and chamber size was not significant in comparison to the changes observed with TOT mice which developed cardiomyopathy without PTU supplementation (Figs. 1 and 2). Confocal microscopy revealed loss of myofibril organization in TOT mice suffering from cardiomyopathy after the withdrawal of PTU in comparison to control nontransgenic mice (Fig. 10, middle). Immunoblot analysis performed with heart samples from PTU-treated TOTs (data not shown) demonstrated that, within $4 \mathrm{~d}$ after withdrawal of PTU, Tmod protein accumulated in TOT mice to levels comparable with those observed in non-PTU-treated TOTs exhibiting symptoms of cardiac failure (Fig. 7).

\section{Discussion}

Dilated cardiomyopathy is the most common form of heart failure (31), yet mechanisms of myocardial damage and compensation are still poorly understood. This report describes a novel model of mouse cardiomyopathy characterized by an interesting phenotype of dilation with a "failed" hypertrophic response. These changes are strikingly similar to those observed by histomorphometric analyses of biopsy samples from human dilated cardiomyopathy $(9,32)$ such as cardiac dilation, thinning of the myocardium relative to chamber area, and enlarged nuclei. TOT mice showed multiple indications of cardiac failure. TOT mice experiencing end-stage heart failure exhibited labored breathing, possibly due to the increased size of the heart making inflation of the lungs difficult. Hearts removed from these mice often contained a thrombus in the left atrium, probably related to decreased filling of the atria due to compromised RV function. Poor pulmonary function accounts for the lack of changes in the lung, since we observed no evidence of pulmonary edema or mononuclear infiltrate (data not shown) as might be expected with RV dilation. Analysis of LV function by Langendorff preparations showed significantly decreased contractility and relaxation in TOT hearts compared with controls. Additionally, on a molecular level, decreased expression of SERCA mRNA is also consistent with cardiac failure (33). These results are consistent with the widespread myofibril degeneration observed throughout the myocardium by fluorescence microscopy. Collectively, these results show the severe cardiomyopathic changes caused by increased Tmod expression. 

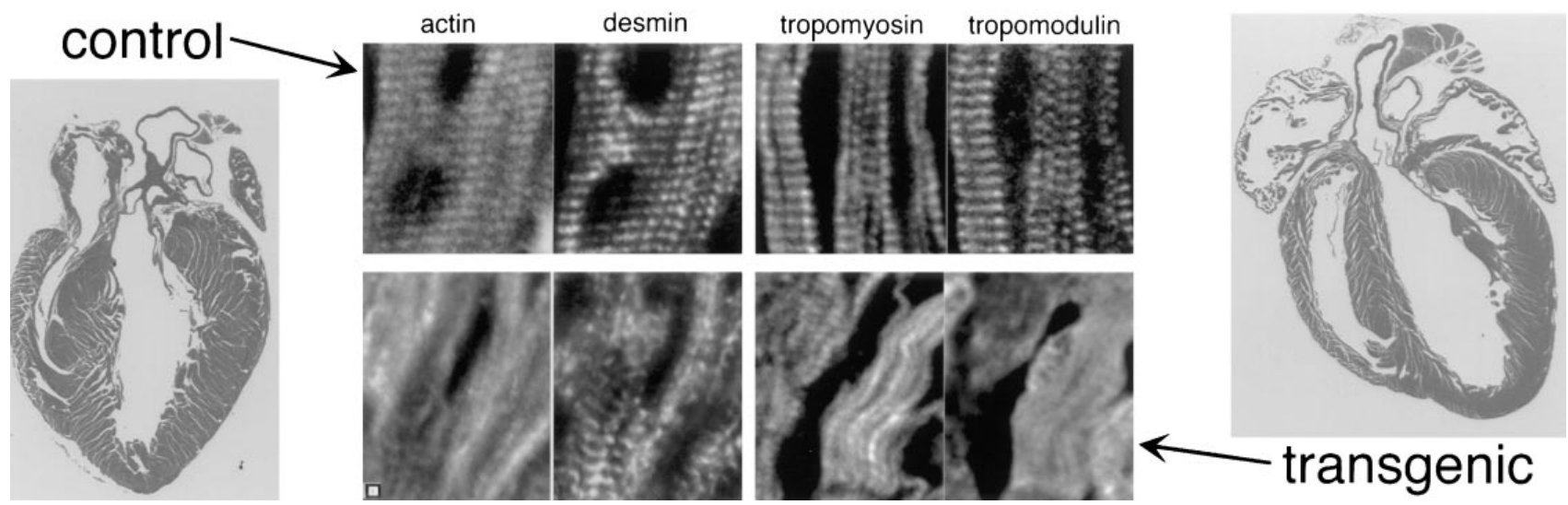

Figure 10. Disruption of myofibril organization in transgenic hearts after withdrawal of PTU. Mice were fed a PTU-supplemented diet from birth for $6 \mathrm{wk}$ before withdrawal. Sections of hearts from mice are shown after withdrawal of PTU supplementation for $4 \mathrm{~d}$. Histologic analysis of heart sections shows overall size and ventricular wall thickness are comparable between nontransgenic control (left) and PTU-treated TOT (right) mice, although atria are larger in the TOT heart. Myofibril structure (middle) in nontransgenic control (top row) and TOT (bottom row) hearts. Antibodies used to label sections are shown at the top of each column. TOT myofibrils show degeneration of thin filaments (shown by disruption of actin and tropomyosin striations) and loss of Tmod organization. Desmin is disrupted as well, although distinct striations can still be found in the TOT heart sample. Bar, $1 \mu \mathrm{m}$.

The pathologic effect of Tmod overexpression in the myocardium was manifested as a loss of myofibril organization (Figs. 4 and 5) which was presumably initiated by deterioration of thin filament structure. Since Tmod is a normal constituent protein of striated muscle, cardiomyocytes tolerate accumulation of the protein without overt toxic effects. In fact, previous experiments with cultured cardiomyocytes showed that Tmod overexpression did not increase metabolic turnover rates for total cellular protein or for the sarcomeric component proteins actin and myosin (15). Thus, Tmod overexpression seems to induce myofibril reorganization rather than protein degradation. This same previous study also demonstrated that the effect of Tmod overproduction upon myofibril structure in the cultured cardiomyocytes was shortened I band width as measured by antiactin antibody and confocal microscopy. Electron microscopy of the cardiomyocytes showed that, despite shortened sarcomeres, remaining thin filaments appeared intact. Collectively, these results of Tmod overexpression in cultured cardiomyocytes suggest that a shorter I band causes a progressive decrease in thin filaments, impairing contractility and leading to degeneration.

The cardiac structural and functional changes in TOT mice are typical for cardiomyopathy resulting from loss of contractile function. Echocardiography showed extreme LV dilatation, wall thinning, and severely depressed LV systolic performance. These features are hallmarks of severe dilated cardiomyopathy in the human. Poor LV performance is partially compensated for by increasing LV cavity size, thereby increasing ventricular preload and moving upwards on the FrankStarling curve. However, severe LV dilatation can result in movement on the downward slope of the curve and even worse ventricular performance. With increasing ventricular dilatation, peak systolic wall stress increases which, in turn, stimulates myocardial thickening to normalize peak wall stress. However, TOT mice appear incapable of an adequate hypertrophic response. In fact, increased expression of atrial natriuretic factor and $\beta$-MHC suggests the heart may activate an incomplete hy- pertrophic response or reactivate embryonic/fetal myosin isoform expression. Hypertrophy results from increases in cell volume coupled with sarcomerogenesis but, since Tmod overexpression leads to myofibril degeneration, the assembly of nascent myofibrils is severely impaired. Thus, myofibril breakdown together with inhibited myofibrillogenesis may act in combination to account for cardiac dilation without substantial hypertrophy. The minimal level of hypertrophy in TOT mice is put into perspective when compared with increased ventricular wall thickness and substantially elevated expression for $\alpha$-skeletal actin found in other experimentally induced mouse cardiomyopathies $(20,34)$.

TOTs in this report are homozygous for the transgene, but the resultant cardiomyopathy is not caused by an insertional effect. Since shutting off Tmod transgene expression with PTU rescued TOTs, the disease stems from $\alpha$-MHC promoter activation in the ventricle after birth coupled with Tmod accumulation. TOT mice maintained on PTU for $6 \mathrm{wk}$ showed no signs of Tmod cardiomyopathy. However, effects of Tmod overexpression appeared within days after switching to a regular (non-PTU) diet. The rapid onset of cardiomyopathic changes in these older TOTs suggests that Tmod protein levels accumulate quickly when the $\alpha$-MHC promoter is fully active. After $4 \mathrm{~d}$ without PTU, cardiomyopathic hearts contained Tmod protein levels comparable with TOTs exhibiting heart failure at 2-4 wk. The relatively minimal remodeling of hearts in PTU-supplemented mice compared with changes observed in normal TOTs is likely due to a rapid accumulation of Tmod in older mice. Apparently, hearts in PTU-supplemented TOT mice had less time to respond when transgene expression was activated and/or was less capable of responding in the older animal.

Many cardiomyopathic conditions share the common characteristic of myofibril degeneration. Myofibril degeneration impairs generation of contractile force resulting in loss of systolic function. Heart diseases with these characteristics such as congestive heart failure or dilated cardiomyopathy have high 
mortality rates. Often, these conditions are ultimately treatable only by transplantation since etiology and pathogenesis are poorly understood. Study of these diseases has been hampered by a lack of suitable experimental animal models to study loss of systolic function due to progressive myofibril degeneration. With this goal, TOT mice were specifically created as an experimental model for progressive myofibril degeneration leading to cardiac failure. TOT mice may serve as a useful model for studying human cardiomyopathic conditions in which there is an inadequate hypertrophic response, such as the cardiomyopathy of anthracycline toxicity (35). In addition to the juvenile onset cardiomyopathy described in this report, other TOT mice express elevated levels of Tmod but live normally and are asymptomatic for external signs of cardiomyopathy. Hearts from these "chronic" TOT mice range in appearance from normal to severely dilated, but Tmod expression is invariably lower than the levels which cause "acute" disease described in this report (our unpublished results). Although initiation of myofibril degeneration by Tmod overexpression is an experimental approach, many features are shared between hearts of TOTs and humans with dilated cardiomyopathy. Experimental studies of TOT mice are likely to provide important insights into the molecular basis of dilated cardiomyopathy, as has been shown for the thick filament-based familial hypertrophic cardiomyopathies $(36,37)$. Construction of a TOT mouse relational database is in progress which will correlate the characteristics of cardiomyopathic TOT hearts with development of disease. Continued characterization of these mice will provide valuable information regarding compensatory, functional, structural, molecular, and physiological changes associated with heart disease.

\section{Acknowledgments}

We thank Jeannie James, Dave Nelson, Steve Schwartz, James Gulick, and Jeff Robbins for helpful discussions. Thanks to Debbie Puthoff for management of the echocardiographic data.

This work was supported by a Grant-in-Aid (American Heart Association, Ohio Affiliate), Scientist Development Grant (American Heart Association, National), and a National Institutes of Health R29 award (HL58224-01) to M.A. Sussman. We also would like to acknowledge support from the Marion-Merrill Dow fund for creating cardiomyopathic transgenic mice, as well as the contribution of Jon Neuman in the creation of our TOTs.

\section{References}

1. Manolio, T.A., K.L. Baughman, R. Rodeheffer, T.A. Pearson, J.D. Bristow, V.V. Michels, W.H. Abelmann, and W.R. Harlan. 1992. Prevalence and etiology of idiopathic dilated cardiomyopathy (summary of a National Heart, Lung, and Blood Institute workshop). Am. J. Cardiol. 69:1458-1466.

2. Ho, K.K., J.L. Pinsky, W.B. Kannel, and D. Levy. 1993. The epidemiology of heart failure: the Framingham study. J. Am. Coll. Cardiol. 22(Suppl. A): 6A-13A.

3. Kleber, F.X., and R. Wensel. 1996. Current guidelines for the treatment of congestive heart failure. Drugs. 51:89-98.

4. Garg, R., M. Packer, B. Pitt, and S. Yusuf. 1993. Heart failure in the 1990s: evolution of a major public health problem in cardiovascular medicine.J. Am. Coll. Cardiol. 22(Suppl. A):3A-5A.

5. Mann, D.L., Y. Urabe, R.L. Kent, S. Vinciguerra, and G. Cooper IV. 1991. Cellular versus myocardial basis for the contractile dysfunction of the hypertrophied myocardium. Circ. Res. 68:402-415.

6. Schaper, J., T.A. Froede, S. Hein, A. Buck, H. Hashizume, B. Speiser, A. Friedl, and N. Bleese. 1991. Impairment of the myocardial ultrastructure and changes of the cytoskeleton in dilated cardiomyopathy. Circulation. 83:504-514.

7. Beltrami, C.A., N. Finato, M. Rocco, G.A. Feruglio, C. Puricelli, E. Cigola, E.H. Sonnenblick, G. Olivetti, and P. Anversa. 1995. The cellular basis of dilated cardiomyopathy in humans. J. Mol. Cell Cardiol. 27:291-305.

8. Marcus, F.I., G.H. Fontaine, G. Guiraudon, R. Frank, J.L. Laurenceau, C. Malergue, and Y. Grosgogeat. 1982. Right ventricular dysplasia: a report of 24 adult cases. Circulation. 65:384-398.

9. Jindal, N., K.K. Talwar, and P. Chopra. 1994. Ultrastructural and histological study of endomyocardial biopsies from patients of dilated cardiomyopathy: a comparative evaluation and their clinical correlation. Indian Heart J. 46: 329-334.

10. Sussman, M.A., S. Sakhi, P. Barrientos, M. Ito, and L. Kedes. 1994. Tropomodulin in rat cardiac muscle. Localization of protein is independent of messenger RNA distribution during myofibrillar development. Circ. Res. 75:221-232.

11. Gregorio, C.C., and V.M. Fowler. 1995. Mechanisms of thin filament assembly in embryonic chick cardiac myocytes. Tropomodulin requires tropomyosin for assembly. J. Cell Biol. 129:683-695.

12. Fowler, V.M., M.A. Sussman, P.G. Miller, B.E. Flucher, and M.P. Daniels. 1993. Tropomodulin is associated with the free (pointed) ends of the thin filaments in rat skeletal muscle. J. Cell Biol. 120:411-420.

13. Fowler, V.M. 1990. Tropomodulin: a cytoskeletal protein that binds to the end of erythrocyte tropomyosin and inhibits tropomyosin binding to actin. J. Cell Biol. 111:471-482.

14. Weber, A.M., C.R. Pennise, G.G. Babcock, and V.M. Fowler. 1994. Tropomodulin caps the pointed ends of actin filaments. J. Cell Biol. 127:16271635 .

15. Sussman, M.A., S. Baque, C.-S. Uhm, M.P. Daniels, B. Price, D. Simpson, L. Terracio, and L. Kedes. 1998. Altered expression of tropomodulin in cardiomyocytes disrupts the sarcomeric structure of myofibrils. Circ. Res. In press.

16. Palermo, J., J. Gulick, M. Colbert, J. Fewell, and J. Robbins. 1996. Transgenic remodeling of the contractile apparatus in the mammalian heart. Circ. Res. 78:504-509.

17. Gulick, J., A. Subramaniam, J. Neumann, and J. Robbins. 1991. Isolation and characterization of the mouse cardiac myosin heavy chain genes. $J$. Biol. Chem. 266:9180-9185.

18. Rindt, H., A. Subramaniam, and J. Robbins. 1995. An in vivo analysis of transcriptional elements in the mouse $\alpha$-myosin heavy chain gene promoter. Transgenic Res. 4:397-405.

19. Ito, M., B. Swanson, M.A. Sussman, L. Kedes, and G. Lyons. 1995 Cloning of tropomodulin cDNA and localization of gene transcripts during mouse embryogenesis. Dev. Biol. 167:317-328.

20. Jones, W.K., I.L. Grupp, T. Doetschmann, G. Grupp, H. Osinska, T.E. Hewett, G. Boivin, J. Gulick, W.A. Ng, and J. Robbins. 1996. Ablation of the murine $\alpha$-myosin heavy chain gene leads to dosage effects and functional deficits in the heart. J. Clin. Invest. 98:1906-1917.

21. Sussman, M.A., E. Battenberg, F.E. Bloom, and V.M. Fowler. 1990. Identification of two nerve growth factor-induced polypeptides in PC12 cells. $J$. Mol. Neurosci. 2:163-174.

22. Sussman, M.A., M. Ito, M.P. Daniels, B. Flucher, S. Buranen, and L. Kedes. 1996. Chicken skeletal muscle tropomodulin: novel localization and characterization. Cell Tissue Res. 285:287-296.

23. Cambon, N., and M.A. Sussman. 1997. Isolation and preparation of single mouse cardiomyocytes for fluorescence confocal microscopy. Methods Cell Sci. 19:83-90.

24. Price, R.L., C. Chintanowonges, I. Shiraishi, T.K. Borg, and L. Terracio. 1996. Local and regional variations in myofibrillar patterns in looping rat hearts. Anat. Rec. 245:83-93.

25. Gulick, J., T.E. Hewett, R. Klevitsky, S.H. Buck, R.L. Moss, and J. Robbins. 1997. Transgenic remodeling of the regulatory myosin light chains in the mammalian heart. Circ. Res. 80:655-664.

26. Jones, W.K., A. Sanchez, and J. Robbins. 1994. Murine pulmonary myocardium: developmental analysis of cardiac gene expression. Dev. Dynam. 200: $117-128$.

27. Maron, B.J., V.J. Ferrans, and W.C. Roberts. 1975. Ultrastructural features of degenerated cardiac muscle cells in patients with cardiac hypertrophy. Am. J. Pathol. 79:387-434.

28. Arber, S., J.J. Hunter, J. Ross, M. Hongo, G. Sansig, J. Borg, J.-C. Perriard, K.R. Chien, and P. Caroni. 1997. MLP-deficient mice exhibit a disruption of cardiac cytoarchitectural organization, dilated cardiomyopathy, and heart failure. Cell. 88:393-403.

29. Tsika, R.W., J.J. Bahl, L.A. Leinwand, and E. Morkin. 1990. Thyroid hormone regulates expression of a transfected human $\alpha$-myosin heavy chain fusion gene in fetal rat heart cells. Proc. Natl. Acad. Sci. USA. 87:379-383.

30. Muthuchamy, M., I.L. Grupp, G. Grupp, B.A. O'Toole, A.B. Kier, G.P. Boivin, J. Neumann, and D.F. Wieczorek. 1995. Molecular and physiological effects of overexpressing striated muscle $\beta$-tropomyosin in the adult murine heart. J. Biol. Chem. 270:30593-30603.

31. Patterson, J.H., and K.F. Adams, Jr. 1996. Pathophysiology of heart failure: changing perceptions. Pharmacotherapy. 16:27S-37S

32. Gallo, P., G. d'Amati, F. Pelliccia, P. Bernucci, C. Cianfrocca, and B. Marino. 1994. Functional significance of myocellular hypertrophy in dilated cardiomyopathy: histomorphometric analysis on 40 endomyocardial biopsies. Am. J. Card. Pathol. 5:11-18.

33. Arai, M., N.R. Alpert, D.H. MacLennan, P. Barton, and M. Periasamy. 
1993. Alterations in sarcoplasmic reticulum gene expression in the human heart failure. A possible mechanism for alterations in systolic and diastolic properties of the failing myocardium. Circ. Res. 72:463-469.

34. Edwards, J.G., G.E. Lyons, B.K. Micales, A. Malhotra, S. Factor, and L.A. Leinwand. 1996. Cardiomyopathy in transgenic myf5 mice. Circ. Res. 78: 379-387.

35. Lipshultz, S.E., S.D. Colan, R.D. Gelber, A.R. Perez-Atayde, S.E. Sallan, and S.P. Sanders. 1991. Late cardiac effects of doxorubicin therapy for acute lymphoblastic leukemia in childhood. N. Engl. J. Med. 324:808-815.

36. Vikstrom, K.L., S.M. Factor, and L.A. Leinwand. 1996. Mice expressing mutant myosin heavy chains are a model for familial hypertrophic cardiomyopathy. Molec. Med. 2:556-567.

37. Geisterfer-Lowrance, A.A.T., M. Christe, D.A. Conner, J.S. Ingwall, F.J. Schoen, C.E. Seidman, and J.G. Seidman. 1996. A mouse model of familial hypertrophic cardiomyopathy. Science. 272:731-734. 